\section{Interferometric spectroscopy}

\author{
E. R. Pike
}

The Principles of Interferometric Spectroscopy. By John Chamberlain. Pp. 347. (Wiley: Chichester, UK, 1979) £20.

THIs book is the result of the completion, collection and revision (to include up-todate advances, by Chantry and Stone) of material left at the time of John Chamberlain's death in 1974. It represents an attempt to bring together the whole field of interferometric spectroscopy at a practical working level in a single self-contained and readily accessible fashion.

As a knowledgeable and able practitioner Chamberlain leads authoritatively from the work of Michelson through a historical introductory chapter to an exposition of introductory theory in chapters 2, 3 and 4. These three chapters cover the ground of the Fourier transform, sampling theory and related topics, the mathematical description of the light field, including the concepts of the analytic signal, coherence and the coherency matrix, and the theory of the practical interference function. The fast Fourier transform is treated in the book's final chapter. Much of this didactic material is, of course, readily available on the bookshelf but as presented here forms a particularly thorough and logical operational approach. A few purist quibbles can be raised here and there as, for example, on page 73 where it is stated that "Physically, the electric and magnetic wave-fields are real quantities ....". Are they? In this book the only signals implicitly used are photon annihilation rates at the detector. If we were to measure an electron detection rate, say, in another branch of physics, would we assert that they derived from a real Schrodinger wave?

The theory of transform spectroscopy forms the core of the text in chapters 5, 6 and 7 . Here are treated, respectively, the measurement of spectral line-shapes from the visibility curves, then non-dispersive and finally dispersive spectroscopy. Again, as in the previous section, the work is notable for its thoroughness and logical development. Full details are given of the effects and practical methods of dealing with them of truncation, sampling and phase errors. Apodisation schemes, for example, are reviewed in considerable detail.

A general information-theoretic approach is absent. The Fourier transform is taken for granted to be the magic requirement to derive what went in from what came out. The method of interferometric spectroscopy, however, is an ill-conditioned first-order Fredholm problem and thus insoluble without a 'model' for the inversion to inject sufficient extra a priori information to remove the ill-conditioning. The Fourier transform, viewed in this light, performs a least-squares fit to a model spectrum consisting of a truncated sum of cosine waves. Although this provides an extremely practical approach to retrieving such information about the spectrum as is transmitted by the instrument it surely does not squeeze out the last drop, and a discussion on these more general lines would have been interesting.

A competent chapter 8 is devoted to various experimental considerations such as finite apertures, imperfect mirrors, modulation schemes and rapid scanning. Perhaps in experimental matters the rival book of R.J. Bell (Introductory Fourier Transform Spectroscopy, Academic Press, 1972) is more extensive.

The importance of interferometric spectroscopy derives in large part from the so-called Fellgett advantage, and it was a disappointment to the reviewer to find, yet again, both in the introductory chapter and in chapter 9 on noise, the commonly propagated fallacious explanation that the improvement is due to seeing all components of the spectrum at once. To a mathematician the nature of a function does not change in its Sturm-Liouville transform and one would naturally ask why the grating is not superior because it sees all delay times at once! This (in his own words) "difficulty in navigating the trickier fine points" shipwrecks him on the erroneous conclusion (page 263) that a multiplex advantage does not exist in the photon noise-limited region, although, apparently without its relevance fully sinking in, the result of Kahn (correct within a factor of 2) is given on page 302 . He founders also on page 299 , getting completely wrong the intensity and photoelectron fluctuations for quasimonochromatic light. "Gaussian" and "Bose-Einstein" should be replaced by "negative-exponential" and "geometric" in this discussion.

Apart from these blemishes, the first of which seems to be indigenous to the subject, the book can be recommended to anyone entering this field, even at the specialist price of $£ 20$.

E.R. Pike is Deputy-Chief Scientific Officer in the Physics Group of the Royal Signals and Radar Establishment, Great Malvern, UK.

\section{Small mammal ecology}

\section{Ian Linn}

Ecology of Small Mammals. Edited by D.M. Stoddart. Pp. 386. (Chapman and Hall: London, 1979.) $£ 15$.

THIS book is an interesting and worthwhile attempt, in a series of essays by specialist authors, to review the state of the art in small mammal ecology and to indicate the areas in which this field of study still requires more intensive investigation. As a review it succeeds quite well. The individual essays reveal, in their expertise, the undoubted skill of the authors in their special fields, and the general coverage makes the whole exercise useful. Nevertheless, there is a lot of bias in this coverage, and it seems to me that the Editor might have exerted more discipline on his contributors, to ensure that the subject was dealt with more evenly.

Thus, the emphasis of the book is firmly laid on the ecology of the rodents of north temperate regions. This emphasis is a simple reflection of the animals and regions about which most is known, but the Editor on the first page of his preface defines small mammals as those weighing less than $5 \mathrm{~kg}$, and there are plenty of insectivores, carnivores and lagomorphs in that category, some smaller primates and even a few artiodactyls. There are honourable mentions of some of these here and there, and a couple of special chapters devoted to bats and marsupials, but more is known than would appear from the text. Even in the case of rodents, which are well covered on the whole, the special problems of tropical animals receive less attention than I would have expected.

If one continues to examine the book with comprehensive coverage in mind, some regrettable general lacunae are to be found. Here I would pick out particularly the topic of behavioural ecology. It is a pity that this aspect of the biology of mammals, which throws such considerable light on their place in the ecosystem, is treated so sparsely. Similarly it is a matter for regret that food habits and feeding ecology are only briefly mentioned.

Perhaps it is too much to expect comprehensiveness in a book of this size, and it should simply be seen as a collection of more or less separate essays, reflecting the views of the authors about certain undoubtedly important areas of knowledge. As such the book is valuable, and can be read with profit by any serious ecologist, and by many others (doctors, veterinarians, pest controllers) whose interest in mammal ecology is more marginal. Professor George Varley once remarked to me that it was time the mammal ecologists got their subject to the same level as the entomologists. Perhaps we still have some way to go, but this book is proof that tremendous strides have been made towards this desirable goal.

Ian Linn is Lecturer in the Department of Biological Sciences at the University of Exeter, UK. 